\title{
Simulated-3D visualisation of artefacts using a portable electromechanical object rig
}

\author{
R. Collmann \\ Art History Program and eScholarship Research Centre \\ University of Melbourne, Australia \\ r.collmann@unimelb.edu.au
}

\author{
A. Borda \\ Victorian eResearch Strategic Initiative \\ Melbourne, Australia \\ ann.borda@versi.edu.au
}

\begin{abstract}
A novel transportable 2-axis electromechanical photographic object rig (Simulated-3D Rig [S3DR] prototype Model 1) was designed and constructed in 2007-08 to allow the inexpensive, noninvasive, time-efficient and high-resolution digital recording and 3D visualisation of cultural artefacts in situ: both within institutional collections and in the field. Experience gained using Model 1 in team-based cross-disciplinary research project use together with project engagement with an Australian State government-funded eResearch body (Victorian eResearch Strategic Initiative, VeRSI) has led to the design and construction of a more versatile S3DR Model 2 incorporating increased functionality, ease-of-use, automation and also stereoscopic photography. The S3DR project thus extended provides a useful example of how collaboration within an eResearch environment can successfully lead to improved visualisation tools and techniques for humanities-based researchers.
\end{abstract}

$3 D$ visualisation. 3D photography. Stereoscopic photography. Archaeology. Art history. Computer visualisation. eResearch. eHumanities. Digital humanities. eScience. Cyberinfrastucture.

\section{INTRODUCTION}

This example of the in-use development and refinement of a previously-existing, well-proven and efficient digital photographic tool, a rotatable 2-axis object rig, provides some insight into how existing (if somewhat older) technologies can be successfully modified in pursuit of optimising specific outputs within particular contextualised practices.

We further hope it provides an interesting example of experience-based need/use tool refinement in modifying a hardware/software visual recording and display apparatus in an environment of evolving technology change.

This continuing and expanding project also provides a useful example of how an eResearch body can energise successful cross-disciplinary, inter-institutional collaboration: in this case, between the Art History Program and the eScholarship Research Centre, University of Melbourne, the History Program, the Archaeology Program and the Department of Physics at La Trobe University, RMIT University and the Australian Institute of Archaeology.

\section{THE ERESEARCH (VERSI) PERSPECTIVE}

VeRSI(http://www.versi.edu.au) was the first Statefunded initiative of its kind in Australia. The establishment of VeRSI follows on the heels of federal recommendations in relation to how Australia, cognisant of efforts elsewhere, such as the United KingdomeScience Programme (http://www.rcuk.ac.uk/escience), could implement a coordinated eResearch plan of core research infrastructures and enabling projects (http://www.dest.gov.au/sectors/research sector/pu blications resources/profiles/e research strat imp framework.htm).

VeRSI was formed in 2006 as a collaborative unincorporated joint venture and comprises the following Consortium members: The University of Melbourne (http://www.unimelb.edu.au),Monash University (http://www.monash.edu.au) and La Trobe University (http://www.latrobe.edu.au) and the Department of Primary Industries (http://www.dpi.vic.gov.au), and with an affiliated member, the Australian government Synchrotron (http://www.synchrotron.gov.au). 
The VeRSI Programme aims to:

(i) accelerate and coordinate the uptake of eResearch in universities, government departments and research organisations within the State of Victoria;

(ii) enable research practitioners to embed the advanced use of ICT in their research and teaching practices;

(iii) inform, through eResearch exemplars, other programmes in Australia and abroad;

(iv) facilitate collaboration across institutions and traditional discipline boundaries.

A principal component of the VeRSI programme is comprised of working with selected research groups across the members to develop use cases illustrating the benefits of eResearch to researchers, primarily in the areas of life sciences and eco-sciences - with a recent growth in other disciplines, such as the humanities. The S3DR project is one of the earliest of the VeRSI humanities engagements.

This project also represents an important intersection between the humanities and science disciplines that characterises a key goal of eResearch, namely to foster cross-disciplinary research and methods. The 3D visualisation of real-world objects, extracted from images, for example, has equal application to cultural heritage research as it does to engineering science (Taubin, 2003).

The extent of this applicability, however, still remains relatively unexplored for humanities, despite the fact that advances in 3D technologies offer new opportunities to record cultural artefacts with higher precision (Hess, 2008), in every detail, and with the ability to 'examine' without compromising the fragile and often impermanent nature of these objects.

The VeRSI Committee selected the S3DR project for funding support due to these possibilities and in recognition that it is not only the new imaging methods that can improve the documentation and preservation of cultural heritage, but innovations in instrumentation, such as the $3 \mathrm{D}$ imaging rig, that can potentially provide more accurate, point-wise measurements of diverse physical properties of objects. Combination of such measurements, 3D imaging and mapping provide a field for the development of new ways to register and present information that can significantly advance the documentation and research of physical objects across the cultural sector, as well as in other subject domains in a true cross-disciplinary eResearch sense.

\section{PROJECT HISTORY}

The original motive for the development of the transportable S3DR came from the rediscovery in 2003 in the Harry Brookes Allen Museum of Anatomy and Pathology collection (http://www.anatomy.unimelb.edu.au/museum), at the University of Melbourne, of a plaster head-cast taken from the notorious and sometimes celebrated 19th century Australian bushranger, Edward (Ned) Kelly after he was executed by hanging in 1880. Head-casts were taken and anatomical parts (particularly the brain) of State-executed criminals were retained post-mortem in Australia and elsewhere in the world until the late 19th century.

In the early to mid 19th century, these physical records were taken for phrenological research purposes, however by the late 19th century, this quasi-science had been largely discredited; the underlying reasons for these practices had been forgotten; the recording and keeping was absorbed by reason of habit into accepted routine procedure: as just a part of the workflow within the State criminal justice execution process. This retentionby-habit is fortunate for the present-day crossdisciplinary historian.

The first-named author wished to use the head cast as the pivotal focus for cross-disciplinary undergraduate teaching purposes with contributions from History of Science, Australian Colonial History, Sociological and Criminological perspectives. It was considered by the subject contributors that such a cross-disciplinary teaching module could well benefit from a web-based multimedia approach. The 3D digital representation of this focal artefact, together with the ability to hotspot (and hyperlink to elucidating information) salient loci of interest on the surface of the 3D representation was the most important criterion for all contributors.

The known and proven technique of usermanipulable computer screen-based V3D using a digital camera mounted on a mechanical rotatable object rig with V3D image creation using Apple QuickTime Virtual Reality (QTVR) stitching software was the only feasible means, at the time, to achieve this purpose.

In general, real and simulated 3D visual experience in humans (and other animals) exploit biologically evolved mechanisms of monocular and binocular visual processing conducted in stages along the visual pathway within the brain (Kandel, 2008, passim). 
The simulated 3D visual experience, whether artificially virtualised or photographically visualised also involves (to varying extents) immersion in the experience partly by commanding brain-mediated 'attention'.

Most 3D digital photographic object rigs developed since the early 1990 s including the one example available in 2003 at the University of Melbourne are large-scale static rigs requiring reticulated electrical power and surrounded by even larger amounts of physical space (at least $10 \times 10 \times 5 \mathrm{~m}$ ) to allow for the servicing of mounted artefacts and rig maintenance access. With most of the artefacts of interest located within geographically widely-distributed institutional collections or in-field it became obvious that there was a need for a portable, self-contained photographic object rig to be taken to objects, rather than vice versa.

\section{METHODS}

The artefact of interest is mounted on a platter that rotates in the horizontal plane while an armmounted camera moves over it in the perpendicular (vertical) plane. Rotational movement in each plane is controlled independently in prior-selected radial increments. The selection of degree increment defines the number of stop points at which the camera shutter is automatically activated. To allow smooth object rotation, increments of 5 degrees in each plane (for instance of 360 degrees horizontal $\times 90$ degrees vertical) result in 1296 images. These separate images are stitched together and hotspots inserted and hyperlinked using the software described below and the resulting fully user-manipulable (rotate, zoom, activate hotspots) .MOV file is displayed on a computer monitor. The file can be web delivered using the many means developed over the last two decades. Experiments in automating the backdrop masking and editing of the many individual images are in progress.

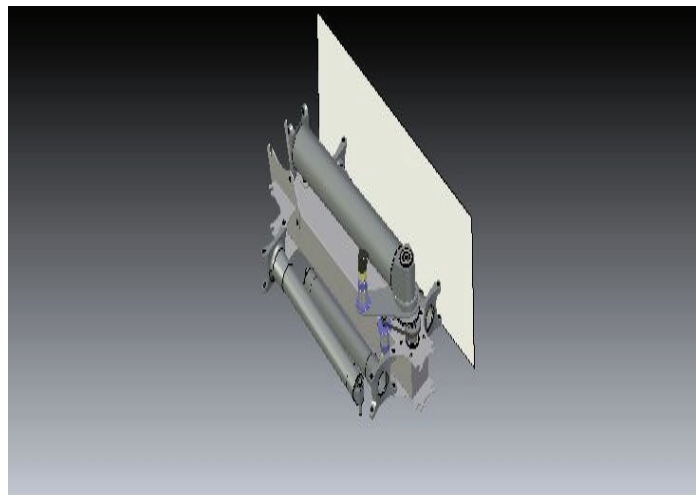

Figure 1:S3DR prototype model 1 - rig collapsed

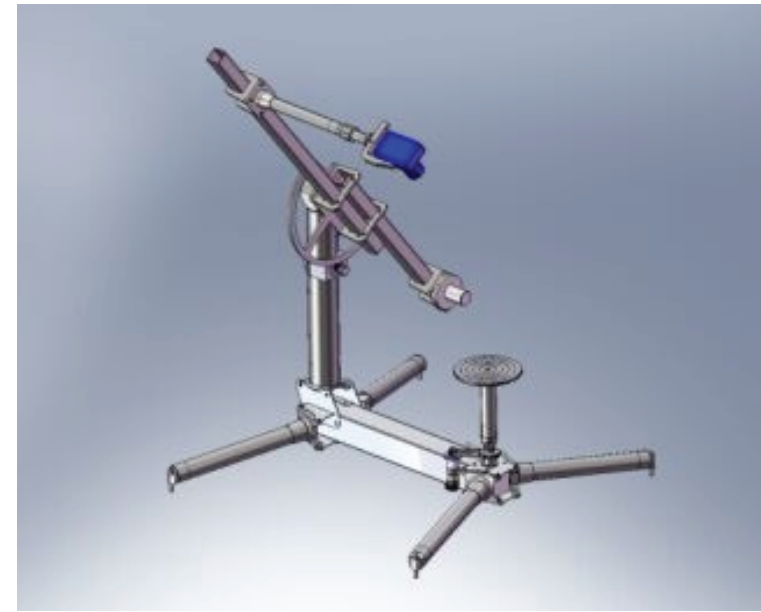

Figure 2:S3DR prototype model 1 - rig extended

Funding for construction of the prototype S3DR Model 1 was successfully requested from the Helen Macpherson Smith Trust, Melbourne, in 2007 (http://hmstrust.org.au).

Prototype model 1 was designed by the first author together with Eric Huwald, Department of Physics, La Trobe University, Bundoora, Victoria, who produced detailed CAD component drawings using SolidWorks (http://www.solidworks.com). Prototype model 1 was designed to be collapsible to allow accommodation in a medium-sized saloon car boot, with dimensions $0.3 \times 0.3 \times 1 \mathrm{~m}$ (collapsed) and $2 \times$ $1 \times 1 \mathrm{~m}$ (expanded) - see figures 1 and 2 . Mass is $\sim 30 \mathrm{Kg}$.

2-axis rig motion is controlled by customised Galil software (http://www.galilmc.com) running on a cable-connected Windows XP laptop computer using a LabVIEW (http://www.ni.com/labview) user interface.

Fourth-year Mechanical and Manufacturing Engineering students from the University of Melbourne sourced all mechanical and electronic components, briefed Whiteforest Engineering (Bundoora, Victoria) on aluminium stock and machining requirements and assembled and displayed the working model 1 as part of a finalyear course project.

\section{IMPLEMENTATION}

3DR Model 1 was successfully trialled and the utility of both portability and good imaging results demonstrated to a variety of potential user audiences in late2008. Somewhat unexpected by the S3DR team, the primary benefit perceived by all audiences was the considerable potential gain in image capture time-efficiency made possible by not 
only the ability to transport the rig to individual collections of many objects but by the subsequent adoption of a serial imaging workflow production line based on categorising artefacts by physical size and depth of relief features and adopting similar focal and lighting measurements for all artefacts within these defined categories.

For the last nine months model 1 has been deployed at the Australian Institute of Archaeology (http://www.aiarch.org.au) where experimental testing using a variety of techniques of image stitching, physical artefact mounting, lighting and digital photography of mass- colour- and volumediverse objects - cuneiform tablets (Figure 3 ) and shabti amongst them - have led to the discovery of some rig limitations and therefore to design modifications and functional enhancements for a more universalmodel 2 (Figure 4). As many of these modifications as possible will be retrofitted to model 1.

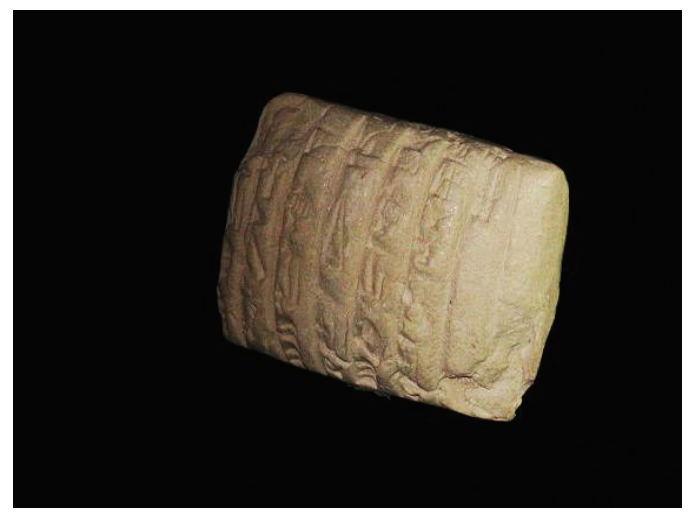

Figure 3:2D image (one of 1296) of Sumerian cuneiform tablet c. 3000BCE taken from final 3D .MOV file

Prototype model 2 funding was secured through VeRSI and the Office of eResearch, La Trobe University. The design team is as for Model 1. All phases of mechanical construction have been undertaken by Whiteforest Engineering. Electronic construction and integration have been performed by Eric Huwald.

In addition to displacing some mechanical components further from the visual vicinity of the mounted object to allow greater flexibility in photographic angles, model 2 will also incorporate stereoscopic photography using an automaticallyactuated (on commanded stop-point camera shutter release) servo-equipped trapezium single camera mounting. The possible choice of using two cameras mounted side-by-side was rejected for cost reasons and that of using recently commercially-available digital stereoscopic domestic model twin-lens cameras was rejected for lack of available current resolution capacity.

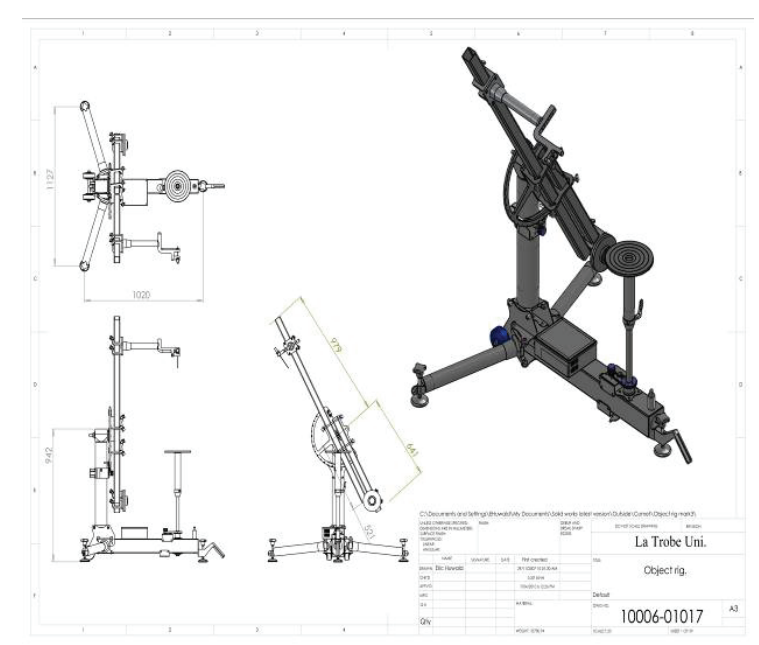

Figure 4: S3DR prototype model 2

Dual simultaneously-rotating stereoscopic usermanipulable V3D .MOV images can then be created and monitor-displayed using Object 2VR software (http://gardengnomesoftware.com) which has now replaced Apple QTVR in project practice. Binocular stereo viewing of the two synchronouslyrotating object images will require colour anaglyph or polarised stereo glasses (most known stereo viewing modes will be supported on our website).Stereo glasses or special-purpose 3D monitors would therefore be required to visualise the hybrid image, although the high-resolution .MOV images will still be available without either. Experiments with 3D LCD/LED screens are currently underway.

The entrainment of binocular visual pathway cues via stereoscopic presentation, in addition to the monocular cues, allows a more immersive experience and has benefit for research and teaching as well as possible therapeutic use with individuals with various forms of cognitive impairment.

\section{FURTHER DEVELOPMENTS}

(i) Camera selection: good results have been obtained with Canon G10 and Canon G11 compact digital cameras. A Nikon D90 DSLR has produced higher resolution images. Optimal lens selection is complex to determine theoretically and has been found to be based on empirical trials. Further improvements in digital camera technology will be incorporated as project time and future funding permits.

(ii) Co-location of a time-of-flight laser with the digital camera for timebased topographic recording purposes for in-field 
archaeological/architectural use. Accurate geometric knowledge of the spatial relationship between the camera and laser positions will allow precise superimposition of surface laser point-cloud information and photographic image pixels to provide accurate Cartesian 3-space information, leading to increased metrical accuracy. This cross-modal superimposition technique, which has been difficult to achieve in the past, could lead to similar superimpositions with other 3D modality point-cloud data output, such as 3D CT, with medical/surgical applications.

(iii) Remote instrumentation: Wireless networked remote-controlled cameras (aperture, focus, shutter release) could be mounted on the S3DR camera arm (Schmidt, 2003). Apart from the need for manual artefact mounting and positioning, these advanced networked tools might lead to outcomes for in-field use similar to those of the Virtual Beam Line at the Australian Synchrotron

(https://www.versi.edu.au/versiprojects/collaborative-

cyberinfrastructure/vbl).

(iv) Collaborative research display of high-resolution manipulable 3D images on OptiPortals worldwide, such as the large Optiportal at the University of Melbourne. This consists of an $8 \times 3$ array of LCD screens with a total resolution over 24 screens of 96 Megapixels (MP). This compares to lecture theatre displays using a light projector of $\sim 1.2 \mathrm{MP}$.

(v) Forensic medicine: The original Ned Kelly plaster head cast will be aligned and matched with a virtual facial reconstruction made from a previously-unknown recently discovered putative Kelly skull. This entirely non-invasive laser and photographic technique could provide strong evidence towards establishing the veracity of the rediscovered skull.

(vi) V3D displays on high-definition OptiPortal / Visualisation Walls (matrix arrays of high-definition, thinbezel LCD/LED/Plasma screens which can reproduce up to 100 megapixel resolution images on wall-sized scales. This tool produces sometimes surprising immersivity by allowing the viewer to move forward (physically approach) 'into' details of interest whilst retaining the 'gestalt' large-scale visualisation in noncentral peripheral visual fields, an effect not found by expanding the magnification of (zooming) areas of interest of images on a smaller-scale desktop monitor.

\section{CONCLUSIONS}

It would appear that, despite some capability limitations, a high-resolution digital cameraequipped portable electromechanical object rig remains a very time efficient, relatively low-cost and short production-time solution to simulated-3D visualisation of smaller-sized artefacts for research and teaching.

The engagement with VeRSI in this project has resulted in a considerable expansion and evolution of the original project and has further led the original S3DR team to become aware of and to use many internet-based collaborativeintercommunication ICT tools not previously available to our team. The S3DR team has found VeRSI to be an expert eResearch research and development group which has been invaluable in expanding the awareness of our specialised yet cross-disciplinary-useful 3D visualisation tool to a wider, global community than we alone could have otherwise had access. A website (http://www.versi.edu.au/3d) and a Sakaiplatformed 3D multimodal discussion group have been constructed and subsequently employed worldwide - exercises beyond the capacity of the original project team.

It is apparent that visualisation tools such as the S3DR, especially when used in large number artefact collections, have the ability to produce large data-sets from similar object categories in this yet not-quite-common V3D form.

We hopeit will therefore rapidly become an instrument which will add much information to the 'data deluge' for which even newer tools, currently under development, will provide productive data access, data mining and thus might provide emergent, creative data analysis.

\section{PROJECT WEBSITE}

This paper, together with all CAD design files, component specifications and sources as well as a library of many digital files of artefacts produced by our team are available at: Simulated-3d visualisation project,http://www.versi.edu.au/3d. 


\section{ACKNOWLEDGEMENTS}

Each author would like to thank the other. In addition, both would like to thank:

The Helen Macpherson Smith Trust

Eric Huwald, Department of Physics, La Trobe University

Dr Lyle Winton, VeRSI

Dr Mark Kosten, eResearch Director, La Trobe

University

Christopher Davey, Director, Australian Institute of Archaeology

Gavan McCarthy, Director, eScholarship Research Centre, University of Melbourne

Joe Arthur, Manager, University Digitisation

Service, University of Melbourne

Dr Armin Schmidt, Archaeological Geophysics, University of Bradford

Professor Malcolm McCormick, RMIT University

Dr Alison Inglis, Art History Program, University of Melbourne

Dr Lisa Beaven, History Program, La Trobe

University

Professor Tim Murray, Head, Archaeology
Program, La Trobe University

David Adam, Teaching, Learning and Research

Support, University of Melbourne

\section{REFERENCES}

Hess, M. (2008) 3D colour scans for object assessment. In EVA London 2008 Conference Proceedings, London, 22-24 July 2008, pp. 125134. British Computer Society.

Kandel, E. and Schwartz, J. (2008) Constructing the visual image. In Kandel, E. and Wurtz, R. (eds.), Principles of Neural Science, 5th edition. McGraw-Hill.

Schmidt, A. (2003) Remote sensing and geophysical prospection. Internet Archaeology, 15. http://intarch.ac.uk/journal/issue15/schmidt index.html (22 April 2010).

Taubin, G. (2002) Building a digital model of Michelangelo's Florentine Pieta. IEEE Computer Graphics and Applications, 22:1, pp. 59-67. 